\title{
Microtearing modes in tokamak discharges
}

\author{
T. Rafiq, ${ }^{1}$ J. Weiland, ${ }^{2}$ A. H. Kritz, ${ }^{1}$ L. Luo, ${ }^{3}$ and A. Y. Pankin ${ }^{4}$ \\ ${ }^{1}$ Department of Physics, Lehigh University, Bethlehem, Pennsylvania 18015, USA \\ ${ }^{2}$ Department of Applied Physics, Chalmers University, S41296 Gothenburg, Sweden \\ ${ }^{3}$ IBM Research, Oak Ridge, Tennessee 37831, USA \\ ${ }^{4}$ Tech-X Corporation, Boulder, Colorado 80308, USA
}

(Received 16 May 2016; accepted 27 May 2016; published online 14 June 2016)

\begin{abstract}
Microtearing modes (MTMs) have been identified as a source of significant electron thermal transport in tokamak discharges. In order to describe the evolution of these discharges, it is necessary to improve the prediction of electron thermal transport. This can be accomplished by utilizing a model for transport driven by MTMs in whole device predictive modeling codes. The objective of this paper is to develop the dispersion relation that governs the MTM driven transport. A unified fluid/kinetic approach is used in the development of a nonlinear dispersion relation for MTMs. The derivation includes the effects of electrostatic and magnetic fluctuations, arbitrary electron-ion collisionality, electron temperature and density gradients, magnetic curvature, and the effects associated with the parallel propagation vector. An iterative nonlinear approach is used to calculate the distribution function employed in obtaining the nonlinear parallel current and the nonlinear dispersion relation. The third order nonlinear effects in magnetic fluctuations are included, and the influence of third order effects on a multi-wave system is considered. An envelope equation for the nonlinear microtearing modes in the collision dominant limit is introduced in order to obtain the saturation level. In the limit that the mode amplitude does not vary along the field line, slab geometry, and strong collisionality, the fluid dispersion relation for nonlinear microtearing modes is found to agree with the kinetic dispersion relation. Published by AIP Publishing.

[http://dx.doi.org/10.1063/1.4953609]
\end{abstract}

\section{INTRODUCTION}

Micro-instabilities can result in turbulence that influences energy confinement in tokamak discharges. One such micro-instability is the microtearing mode (MTM), a tearing-parity mode centered on high-order rational surfaces. Microtearing instability can provide a significant contribution to the electron thermal transport in low-aspect ratio tokamaks. ${ }^{1-5}$ The MTMs lead to a tearing and subsequent reconnection of the magnetic field. MTMs are shortwavelength ion scale (low $k_{\theta}$ ) electromagnetic instabilities that are driven by electron temperature gradients. ${ }^{6-8}$ It was proposed that when the magnetic field has a component in the same direction as the electron temperature gradient, a current is driven in the direction of the magnetic field line, which can destabilize MTMs. These modes propagate in the electron diamagnetic drift direction and depend on the electron ion collisionality. ${ }^{9,10}$ Consequently, transport driven by MTM instabilities depends on both the electron ion collision frequency and the electron temperature gradient. The research carried out in this paper indicates that when the electrostatic effects are included, MTMs also depend on the density gradient.

It has been shown that for some tokamak discharges, MTMs are unstable primarily in the plasma edge region, ${ }^{11,12}$ regulating heat transport, and, possibly, pedestal evolution between edge-localized modes. ${ }^{9}$ Moreover, it has been found that in plasmas where $\beta_{e}$ (ratio of the electron pressure to the magnetic pressure) and collisionality are sufficiently high, MTMs can become the dominant instability contributing to electron transport in the plasma core. ${ }^{13}$ MTMs may be unstable even when collisionality is negligible with the role of collisionality replaced by particle inertia. ${ }^{14}$

Unstable microtearing modes occur in the interior of NSTX as a consequence of high electron ion collisionality associated with low electron temperature and high electron density. ${ }^{3,5}$ In a GS2 gyrokinetic stability analysis, ${ }^{1}$ microtearing modes were found to be the most unstable modes in the NSTX radial region $0.4 \leq r / a \leq 0.75$. Non-linear gyrokinetic GYRO code calculations have shown that microtearing modes in high $\beta \mathrm{H}$-mode NSTX-U plasmas can drive electron transport at levels that match those inferred from the experimental data. ${ }^{4}$ Moreover, the simulations are found to yield a result that is consistent with the collisionality scaling that is observed in the NSTX discharges.

The frequency of MTMs is generally greater than the electron diamagnetic drift frequency. MTMs, sometimes referred to as drift-tearing modes, have a mode structure that is extended along the magnetic field lines. The perturbed magnetic field associated with MTMs has even parity, and there are magnetic islands near rational magnetic surfaces. It is found that MTMs drive electron heat transport through magnetic potential fluctuations, while contributions associated with electrostatic perturbations and compressional magnetic perturbations are typically smaller. Ion thermal and particle transport driven by MTMs are negligible. ${ }^{13}$

In order to understand how MTMs affect transport and consequently the evolution of electron temperature in tokamak discharges, a reduced transport model for microtearing modes is needed for use in integrated predictive modeling. ${ }^{15}$ The derivation of the MTM model requires the development 
of a nonlinear dispersion relation for MTMs. In this paper, the derivation of the dispersion relations begins with an approach that utilizes the linear gyrokinetic equation for electrons in which there is no constraint on the magnitude of the collision frequency relative to the mode frequency. The collision frequency dependence on velocity is expressed in terms of the electron thermal velocity.

To advance the kinetic description and to include nonlinear effects due to magnetic fluctuations, a unified fluid/kinetic approach is utilized to derive the nonlinear dispersion relation. The electron momentum, electron density, Maxwell equations, Ampere's law, and quasi-neutrality condition are used in the derivation. Effects of electrostatic and magnetic fluctuations, collisionality, electron temperature gradient, density gradient, arbitrary curvature, and the physics associated with parallel propagation vector are included. An iterative approach is used to calculate the nonlinear distribution function, which, in turn, is used to calculate nonlinear parallel current in order to obtain nonlinear dispersion relation. The influence of third order nonlinear effects on a multiwave system is considered. The third order effects provide a possible mechanism for the temporal saturation of the microtearing instability. An envelope equation for the evolution of the nonlinear microtearing modes in the collision dominant limit is introduced. It is shown that in the slab geometry and strong collisionality limit, when the mode amplitude does not vary along the field line, the fluid dispersion relation for nonlinear microtearing modes agrees with the kinetic dispersion relation of Ref. 16 .

The paper is organized as follows. In Sec. II, electron gyrokinetic equation with magnetic drift due to bending of field lines is used to calculate the linear dispersion relation of microtearing modes in the limit that the mode amplitude does not vary along the field lines. In Sec. III, the nonlinear fluid equations for the microtearing modes are presented. In Sec. IV, the nonlinear parallel current perturbation is calculated using an iterative nonlinear approach. The third order nonlinear effects in magnetic fluctuations are included in the development of microtearing mode dispersion relation, and the influence of third order effects on a multi-wave system is considered. In Sec. V, the nonlinear microtearing dispersion relations are derived and an envelope equation for the evolution of the nonlinear microtearing modes is obtained in the collision dominant limit. The fluid/kinetic approach for developing the nonlinear MTM dispersion relation is summarized in Sec. VI.

\section{DERIVATION OF LINEAR MTM DISPERSION RELATION}

The physics of microtearing modes is associated with the electron dynamics along the direction of the magnetic field. The electron gyrokinetic equation, including magnetic drift due to the bending of field lines, is (Eq. (5.28) of Ref. 17)

$$
\begin{aligned}
& \left(\omega-k_{\|} u_{\|}-\omega_{\operatorname{De}}\left(u_{\|}^{2}, u_{\perp}^{2}\right)-u_{\|} \frac{\delta \mathbf{B}}{B} \cdot \mathbf{k}+i \nu(u)\right)\left(f_{k}-\frac{e \phi}{T_{\mathrm{e}}} f_{0}\right) \\
& =-\frac{e}{T_{\mathrm{e}}}\left(\omega-\omega_{* \mathrm{e}}(u)\right) \times\left(\phi-u_{\|} A_{\|}\right) f_{0} .
\end{aligned}
$$

The distribution function for describing parallel electron motion is

$$
\begin{aligned}
f_{k}= & \frac{e \phi}{T_{\mathrm{e}}} f_{0}-\left(\frac{e \phi}{T_{\mathrm{e}}}-u_{\|} \frac{e A_{\|}}{T_{\mathrm{e}}}\right) \\
& \times \frac{\omega-\omega_{* \mathrm{e}}(u)}{\omega-\omega_{\operatorname{De}}\left(u_{\|}^{2}, u_{\perp}^{2}\right)-k_{\|} u_{\|}+i \nu(u)} f_{0} \\
& +\frac{u_{\|} \frac{\delta \mathbf{B}}{B} \cdot \mathbf{k}}{\omega-\omega_{\operatorname{De}}\left(u_{\|}^{2}, u_{\perp}^{2}\right)-k_{\|} u_{\|}+i \nu(u)} f_{k} .
\end{aligned}
$$

In the computation of the parallel current in this section, the last term on the right in Eq. (2) is neglected. However, the last term on the right results in the equation for $f_{k}$ being nonlinear and in Sec. IV, the solution for $f_{k}$ is obtained iteratively.

The perturbed electron parallel current $j_{\|}$, with the kinetic effects driven by an electron temperature gradient, can lead to instability of the microtearing mode in a collisional magnetized plasma. The parallel current, $j_{\|}$, is obtained by including only even powers of parallel velocity $u_{\|}$

$$
\begin{aligned}
j_{\|} & =-\int e f_{k} u_{\|} d u_{\|} \\
& =-\frac{e^{2} A_{\|}}{T_{e}} \int u_{\|}^{2} \frac{\omega-\omega_{* e}\left[1+\eta_{e}\left(\frac{m u^{2}}{2 T_{\mathrm{e}}}-\frac{3}{2}\right)\right]}{\omega-\omega_{\operatorname{De}}\left(u_{\|}^{2}, u_{\perp}^{2}\right)-k_{\|} u_{\|}+i \nu(u)} f_{0} d u_{\|} .
\end{aligned}
$$

The subscript $\|$ refers to the component of a vector along $\hat{b} \equiv \mathbf{B} /|B|$, e.g., $\mathbf{u}_{\|} \equiv \hat{b} \hat{b} \cdot \mathbf{u}$, and $\mathbf{u}_{\perp} \equiv \mathbf{u}-\mathbf{u}_{\|} . \mathbf{B}$ is the magnetic field, $\mathbf{u}$ is the electron velocity, $\delta B$ is the perturbed magnetic field, and $f_{0}$ is the Maxwellian distribution function. The integration in Eq. (3) is carried out by utilizing the approach employed in Ref. 18. The zero order and first order results for $j_{\|}$can be obtained by initially ignoring collisions and then improving this result with the $\eta_{e}$ part of the integrand so that $j_{\|}=j_{\| 0}+j_{\| 1}$ where

$$
j_{\| 0}=-\varepsilon_{0} \omega_{\mathrm{pe}}^{2} \frac{\omega-\omega_{* \mathrm{e}}\left(1+\eta_{e}\right)}{\omega-\omega_{\mathrm{De}}} A_{\|},
$$

and

$$
j_{\| 1}=-\alpha_{0} \varepsilon_{0} \omega_{\mathrm{pe}}^{2} \frac{\eta_{e} \omega_{* \mathrm{e}}}{\omega-\omega_{\mathrm{De}}+i \nu_{\mathrm{ei}}} A_{\|} .
$$

In the $j_{\| 0}$ and $j_{\| 1}$ calculations, the parallel propagation vector $k_{\|}$is ignored. However, later it will be included using a fluid approach. In Eq. (5), $\alpha_{0}$ is a free parameter introduced to compensate for the velocity dependence of the collision frequency $\nu(u)$. The $\omega_{p e} \equiv \sqrt{n e^{2} / \epsilon_{0} m_{e}}$ is the electron plasma frequency, $n$ is the electron density, $e$ is the electron charge, $\varepsilon_{0}$ is the permittivity of free space, and $m_{e}$ is the electron mass. The electron diamagnetic drift frequency is given by

$$
\begin{aligned}
\omega_{* \mathrm{e}}(u) & =\omega_{* \mathrm{e}}\left[1+\eta_{\mathrm{e}}\left(\frac{m u^{2}}{2 T_{\mathrm{e}}}-\frac{3}{2}\right)\right], \text { where } \\
\omega_{* e} & =\frac{\mathrm{k}_{\mathrm{y}} \mathrm{T}_{\mathrm{e}} \mathrm{g}_{\mathrm{ne}}}{\mathrm{eBR}},
\end{aligned}
$$


and where $k_{y}$ is the wave vector in the poloidal direction, $T_{e}$ is the electron temperature, $g_{\mathrm{ne}} \equiv-R \hat{\mathbf{x}} \cdot \nabla n_{e} / n_{e}, R$ is the major radius to geometric center of the flux surface, $\hat{\mathbf{x}}$ is the unit vector in the radial direction, and $\mathbf{A}_{\|}$is the parallel magnetic vector potential.

The ratio of electron temperature and electron density gradient is

$$
\eta_{e} \equiv g_{\mathrm{Te}} / g_{\mathrm{ne}}=\frac{n_{e} \hat{\mathbf{x}} \cdot \nabla T_{e}}{T_{e} \hat{\mathbf{x}} \cdot \nabla n_{e}}
$$

The electron diamagnetic drift frequency is $\omega_{\text {De }}=\mathbf{k} \cdot \mathbf{u}_{\text {De }}$, where $\mathbf{u}_{\mathrm{De}}$ is the drift velocity due to $\nabla|B|$ and magnetic field curvature, $\kappa=\hat{\mathbf{b}} \cdot \nabla \hat{b}$, and is given by

$$
\mathbf{u}_{D e}=\frac{T_{e}}{m_{e} \Omega_{i}} \hat{\mathbf{b}} \times\left(\frac{\nabla B}{B}+\kappa\right) .
$$

The electron collision frequency $\nu_{\mathrm{ei}}$ is given by

$$
\nu_{\mathrm{ei}}=4(2 \pi)^{1 / 2} n_{e}(\ln \lambda) e^{4} Z_{\mathrm{eff}} /\left[3\left(4 \pi \epsilon_{o}\right)^{2} m_{e}^{1 / 2}\left(k_{b} T_{e}\right)^{3 / 2}\right],
$$

where $\lambda$ is the Coulomb logarithm, $Z_{\mathrm{eff}}$ is the effective charge, and $k_{b}$ is the Boltzmann constant.

The linear dispersion can be obtained by equating $j_{\|}$ given by the Ampéres law

$$
j_{\|}=\frac{1}{\mu_{0}} k_{\perp}^{2} A_{\|}
$$

with $j_{\|}$given by the sum of Eqs. (4) and (5)

$$
\begin{aligned}
\omega\left(1+k_{\perp}^{2} c^{2} / \omega_{\mathrm{pe}}^{2}\right)= & \omega_{* \mathrm{e}}\left(1+\eta_{e}\right)-\alpha_{0} \eta_{\mathrm{e}} \omega_{* \mathrm{e}} \frac{\omega-\omega_{\mathrm{De}}}{\omega-\omega_{\mathrm{De}}+i \nu_{\mathrm{ei}}} \\
& +\left(k_{\perp}^{2} c^{2} / \omega_{\mathrm{pe}}^{2}\right) \omega_{\mathrm{De}} .
\end{aligned}
$$

Ignoring $\omega_{\mathrm{De}}, k_{\perp}^{2} c^{2} / \omega_{\mathrm{pe}}^{2}$ and taking collision dominant limit in Eq. (9), one finds that

$$
\omega=\omega_{* \mathrm{e}}\left(1+\eta_{e}\right)+i \alpha_{0} \eta_{\mathrm{e}} \omega_{* \mathrm{e}} \frac{\omega}{\nu_{\mathrm{ei}}} .
$$

The electron temperature gradient can induce linear destabilization of microtearing modes in the presence of electron collisionality. The comparison of Eq. (10) with the kinetic equation (8) of Ref. 16 yields the result for $\alpha_{0}$

$$
\alpha_{0}=\frac{3}{8} \Gamma\left(\frac{9}{2}\right)
$$

Note that the value of $\alpha_{0}$ depends on the choice of collision model. Here, the value of $\alpha_{0}$ corresponds to the Lorentz collision model used in Ref. 16.

\section{NONLINEAR FLUID EQUATIONS FOR MICROTEARING MODES}

In order to include nonlinear effects, the parallel electron motion is generalized as shown below

$$
\begin{aligned}
& \frac{\partial u_{\|}}{\partial t}+\nu_{\mathrm{ei}} u_{\|}+u_{\|} \hat{\mathbf{e}}_{\|} \cdot \nabla u_{\|}+\mathbf{u}_{\mathrm{gc}} \cdot \nabla u_{\|} \\
& \quad=\frac{-e}{m_{\mathrm{e}}}\left\{E_{\|}+\left[\left(\mathbf{u}_{E}+\mathbf{u}_{*}\right) \times \delta \mathbf{B}_{\perp}\right] \cdot \hat{\mathbf{e}}_{\|}\right\}-\frac{\hat{\mathbf{e}}_{\|} \cdot \nabla P_{\mathrm{e}}}{m_{\mathrm{e}} n_{\mathrm{e}}},
\end{aligned}
$$

where

$$
\mathbf{u}_{\mathrm{gc}}=\mathbf{u}_{\mathrm{E}}+\mathbf{u}_{\mathrm{De}}+u_{\|} \frac{\delta \mathbf{B}}{B} .
$$

The last term in Eq. (13) represents the nonlinear contribution. The electric field vector, E, and magnetic field vector, $\mathbf{B}$, are given in terms of the scalar potential, $\phi$, and the parallel component of vector potential, $\mathbf{A}_{\|}$

$$
\mathbf{E}=-\nabla \phi-\frac{\partial \mathbf{A}_{\|}}{\partial t}, \quad \mathbf{B}=\nabla \times \mathbf{A}_{\|}
$$

It is assumed here that the coupling with compressional modes is negligible in low- $\beta$ plasmas.

It is necessary to solve for the perturbed density in order to calculate the parallel current

$$
\begin{aligned}
& \left(\frac{\partial}{\partial t}+\mathbf{u}_{\mathrm{E}} \cdot \nabla+\mathbf{u}_{\mathrm{De}} \cdot \nabla\right) \frac{\delta n_{\mathrm{e}}}{n_{e}}+\left(\mathbf{u}_{* \mathrm{e}}-\mathbf{u}_{\mathrm{De}}\right) \cdot \nabla \frac{e \phi}{T_{\mathrm{e}}} \\
& \quad+\mathbf{u}_{\mathrm{De}} \cdot \nabla \frac{\delta T_{\mathrm{e}}}{T_{\mathrm{e}}}-\rho_{\mathrm{s}}^{2} u_{\mathrm{A}}^{2} \frac{\hat{\mathbf{e}}_{\|} \times \nabla A_{\|}}{B_{0}} \cdot \nabla \Delta_{\perp} \frac{e A_{\|}}{T_{\mathrm{e}}} \\
& \quad+\rho_{\mathrm{s}}^{2} u_{\mathrm{A}}^{2} \hat{\mathbf{e}}_{\|} \cdot \nabla \Delta_{\perp} \frac{e A_{\|}}{T_{\mathrm{e}}}=0,
\end{aligned}
$$

where

$$
\mathbf{u}_{E}=\frac{\hat{\mathbf{b}} \times \nabla \phi}{B} \quad \text { and } \quad \mathbf{u}_{* e}=-T_{e} \frac{\hat{\mathbf{b}} \times \nabla n_{e}}{e n_{e} B}=\hat{y} \omega_{* e} / k_{y} .
$$

In Eq. (15), $\rho_{s} \equiv c_{s} / \Omega_{i}$ is the ion Larmor radius with the electron (rather than the ion) temperature, $c_{s} \equiv \sqrt{T_{e} / m_{i}}$ is the speed of sound, $\Omega_{i}=e B / m_{i}$ is the ion gyroradius, $u_{A}=$ $B / \sqrt{\mu_{0} n_{e} m_{i}}$ is the Alfvén velocity, and $\mu_{0}$ is the permeability of free space.

The parallel velocity is obtained by using the component of Ampères law parallel to the magnetic field

$$
u_{\|}=\rho_{\mathrm{s}}^{2} u_{\mathrm{A}}^{2} \frac{e}{T_{\mathrm{e}}} \Delta A_{\|} \cdot
$$

Thus, the leading order nonlinear term in Eq. (12) is

$$
u_{\|} \frac{\delta \mathbf{B}}{B} \cdot \nabla u_{\|}=u_{\|} \rho_{\mathrm{s}}^{2} u_{\mathrm{A}}^{2} \frac{e}{T_{\mathrm{e}}} \frac{\delta \mathbf{B}}{B} \cdot \nabla \Delta A_{\|} \cdot
$$

The microtearing modes are different than the other microinstabilities since the $\delta \mathbf{B}$ fluctuations can result in small scale magnetic islands.

In Sec. IV, the nonlinear parallel current perturbation is calculated, and the influence of third order effects on a multi-wave system is considered.

\section{NONLINEAR PARALLEL CURRENT PERTURBATION}

The parallel current is obtained by combining Eqs. (12) and (15) and retaining only the leading nonlinear term 


$$
\begin{aligned}
\left(\frac{\partial}{\partial t}+\right. & \left.\mathbf{u}_{\mathrm{De}} \cdot \nabla+\nu_{\mathrm{ei}}\right) j_{\|} \\
= & -\frac{e^{2} n}{m_{\mathrm{e}}}\left\{\hat{\mathbf{e}}_{\|} \cdot \nabla \phi+\left(\frac{\partial}{\partial t}+\mathbf{u}_{* \mathrm{e}} \cdot \nabla\right) A_{\|}\right\} \\
& +e n_{\mathrm{e}} \frac{T_{\mathrm{e}}}{m_{\mathrm{e}}} \hat{\mathbf{e}}_{\|} \cdot \nabla\left(\frac{e \phi}{T_{\mathrm{e}}}+\frac{\delta n_{\mathrm{e}}}{n_{\mathrm{e}}}\right) \\
& -e n u_{\|}\left(\mathbf{u}_{* \mathrm{e}}+\mathbf{u}_{\mathrm{De}}\right) \cdot \nabla \frac{e \phi}{T \mathrm{e}}+2 e n u_{\|} \frac{\delta \mathbf{B}}{B} \cdot \nabla u_{\|},
\end{aligned}
$$

where

$$
\frac{\delta n_{\mathrm{e}}}{n_{\mathrm{e}}}=\frac{\omega_{* \mathrm{e}}-\omega_{\mathrm{De}}}{\omega-\omega_{\mathrm{De}}} \frac{e \phi}{T_{\mathrm{e}}} .
$$

The relation between $\phi$ and $A_{\|}$can be found using the Ampére's law and the quasi neutrality condition

$$
\phi \approx \frac{k_{\|} u_{A}^{2}}{\omega} A_{\|}
$$

An equation for the parallel current in $\omega, k$ space can be obtained from Eq. (18) with $\delta n_{e}$ given in Eq. (19) and $\phi$ given in Eq. (20)

$$
\begin{aligned}
j_{\|}= & j_{\| \mathrm{L}}-j_{\| \phi}+\frac{j_{\|}}{\omega-\omega_{\mathrm{De}}+i \nu_{\mathrm{ei}}} \\
& \times\left[\left(\omega_{* \mathrm{e}}-\omega_{\mathrm{De}}\right) \frac{e \phi}{T_{\mathrm{e}}}-2 \frac{\delta \mathbf{B} \cdot \mathbf{k}}{B} \frac{j_{\|}}{e n_{\mathrm{e}}}\right],
\end{aligned}
$$

where

$$
j_{\| \mathrm{L}}=-\epsilon_{0} \omega_{\mathrm{pe}}^{2} \frac{\omega-\omega_{* \mathrm{e}}\left(1+\eta_{\mathrm{e}}\right)}{\omega-\omega_{\mathrm{De}}+i \nu_{\mathrm{ei}}} A_{\|}
$$

and

$$
j_{\| \phi}=-\frac{\varepsilon_{0} \omega_{\mathrm{pe}}^{2}}{\omega-\omega_{\mathrm{De}}+i \nu_{\mathrm{ei}}} \frac{\omega_{* \mathrm{e}}-\omega_{\mathrm{De}}}{\omega-\omega_{\mathrm{De}}} \frac{k_{\|}^{2} u_{\mathrm{A}}^{2}}{\omega} A_{\|} .
$$

Note that $j_{\| \mathrm{L}}$ is the parallel current, $j_{\| 0}$ derived in the gyrokinetic analysis above but modified to include the dependence on collision frequency. In order to maintain the connection with the gyrokinetic approach, in the analysis below $j_{\| \mathrm{L}}$ will be replaced by $j_{\| 0}+j_{\| 1}$ in Eq. (40).

The cubic nonlinear term in Eq. (21) is proportional to

$$
j_{\|} \frac{\delta \mathbf{B} \cdot \mathbf{k}}{B} j_{\|} \cdot
$$

This is the only phase independent term that yields a nonlinear frequency shift. This is needed for saturation of microtearing modes. This term in the absence of a toroidal effect $\left(\omega_{\text {De }}=0\right)$ is of the same type as derived by Drake et al.

In Subsection IV A, an iterative approach will be used to calculate the nonlinear parallel current perturbation. Subsequently, the nonlinear parallel current perturbation will be utilized to calculate nonlinear microtearing dispersion relation.

\section{A. Iterative nonlinear approach for evaluating $\delta f$}

As described above, the cubic nonlinear term impacts the evolution of the nonlinear current. The nonlinear current is a consequence of the nonlinear component of the distribution function that is associated with the parallel electron motion. In Section II, $j_{\| 0}$, based on the linear analysis, and $j_{\| 1}$, the contribution of parallel current that is a consequence of the velocity dependence of collision frequency, were obtained (Eqs. (4) and (5)). Below the contribution to the parallel current, $j_{\| 3}$, resulting from the nonlinear term in Eq. (21), the final term on the right side is obtained

$$
j_{\| 3}=-e \int u_{\|} \delta f d u_{\|} .
$$

The parallel current can be computed once modification of the distribution function, $\delta f$, resulting from the presence of the nonlinear term is obtained.

In order to calculate the nonlinear parallel current $j_{\| 3}$, the distribution function $\delta f$ is calculated iteratively as illustrated below

$$
\delta f_{1}=\frac{e \phi}{T_{\mathrm{e}}} f_{0}-\left(\frac{e \phi}{T_{\mathrm{e}}}-u_{\|} \frac{e A_{\|}}{T_{\mathrm{e}}}\right) \frac{\omega-\omega_{* \mathrm{e}}(u)}{\omega-\omega_{\mathrm{De}}\left(u_{\|}^{2}, u_{\perp}^{2}\right)+i \nu(u)} f_{0} .
$$

In the computation of $\delta f_{1}$, the focus is on the electromagnetic contribution

$$
\delta f_{1} \approx u_{\|} \frac{e A_{\|}}{T_{\mathrm{e}}} \frac{\omega-\omega_{* \mathrm{e}}(u)}{\omega-\omega_{\operatorname{De}}\left(u_{\|}^{2}, u_{\perp}^{2}\right)+i \nu(u)} f_{0} .
$$

The linear correction to $\delta f$, that is, $\delta f_{1}$, does not include the contribution from the nonlinear term. The next iteration in the solution for $\delta f$ yields

$$
\delta f_{2}=\frac{u_{\|} \frac{\delta \mathbf{B}}{B} \cdot \mathbf{k}}{\omega-\omega_{\operatorname{De}}\left(u_{\|}^{2}, u_{\perp}^{2}\right)+i \nu(u)} \delta f_{1},
$$

while the iteration, $\delta f_{2}$, does include a contribution to $\delta f$ associated with the nonlinear term; it does not result in a contribution to $j_{\| 3}$ because the integrand is odd in $u_{\|}$. Thus, proceeding to the next order in iteration, one obtains

$$
\delta f_{3}=\frac{u_{\|} \frac{\delta \mathbf{B}}{B} \cdot \mathbf{k}}{\omega-\omega_{\operatorname{De}}\left(u_{\|}^{2}, u_{\perp}^{2}\right)+i \nu(u)} \delta f_{2} .
$$

Equation (28) does not take into account the phase mixing associated with the turbulence. In order to account for the turbulence phase mixing, we will use the iterative approach described above to obtain the perturbed distribution associated with multi-wave coupling.

\section{B. Perturbed distribution associated with multi-wave coupling}

In a plasma, the main nonlinear effects may be classified as wave-particle interactions and wave-wave interactions. Phase mixed turbulence due to wave-wave interaction is considered in this section. The second order phase dependent nonlinearities are ignored, but the phase independent cubic 
nonlinearity is included. This cubic nonlinearity results in phase independent nonlinear frequency shifts which provide the saturation of the instability.

The contribution to the interaction, from the third order in $\delta B$, is of the four-wave interaction type such that

$$
k=k^{\prime}+k^{\prime \prime}+k^{\prime \prime \prime} \text {. }
$$

Only three-waves are assumed to be present, and, therefore, two waves must be the same. The $\delta f_{3}$ in Eq. (28) is written for an unknown mode number just to indicate which terms will be present. These terms are written in order to produce a $\delta f_{3 k}$ with the mode number $k$ as required. The $\delta f_{3 k}$ will then be used to calculate the component of parallel current due to the third order nonlinear perturbation in parallel velocity and magnetic field combined

$$
\delta f_{3 k}=\frac{u_{\|} \frac{\delta \mathbf{B}_{k^{\prime}}}{B} \cdot\left(\mathbf{k}-\mathbf{k}^{\prime}\right) \delta f_{2\left(k-k^{\prime}\right)}+u_{\|} \frac{\delta \mathbf{B}_{k-k^{\prime}}}{B} \cdot \mathbf{k}^{\prime} \delta f_{2 k^{\prime}}}{\omega-\omega_{\operatorname{De}}\left(u_{\|}^{2}, u_{\perp}^{2}\right)+i \nu(u)} .
$$

Then from Eq. (27), $\delta f_{2\left(k-k^{\prime}\right)}$ and $\delta f_{2 k^{\prime}}$ can be calculated as

$$
\begin{aligned}
& \delta f_{2\left(k-k^{\prime}\right)} \\
& =\frac{u_{\|} \frac{\delta \mathbf{B}_{k^{\prime \prime}}}{B} \cdot\left(\mathbf{k}-\mathbf{k}^{\prime}-\mathbf{k}^{\prime \prime}\right) \delta f_{1\left(k-k^{\prime}-k^{\prime \prime}\right)}+u_{\|} \frac{\delta \mathbf{B}_{k-k^{\prime}-k^{\prime \prime}}}{B} \cdot \mathbf{k}^{\prime \prime} \delta f_{1 k^{\prime \prime}}}{\omega_{k-k^{\prime}}-\omega_{\operatorname{De} k-k^{\prime}}\left(u_{\|}^{2}, u_{\perp}^{2}\right)+i \nu(u)},
\end{aligned}
$$

and

$$
\delta f_{2 k^{\prime}}=\frac{u_{\|} \frac{\delta \mathbf{B}_{k^{\prime \prime}}}{B} \cdot\left(\mathbf{k}^{\prime}-\mathbf{k}^{\prime \prime}\right) \delta f_{1\left(k^{\prime}-k^{\prime \prime}\right)}+u_{\|} \frac{\delta \mathbf{B}_{k^{\prime}-k^{\prime \prime}}}{B} \cdot \mathbf{k}^{\prime \prime} \delta f_{1 k^{\prime \prime}}}{\omega_{k^{\prime}}-\omega_{\operatorname{De} k^{\prime}}\left(u_{\|}^{2}, u_{\perp}^{2}\right)+i \nu(u)} .
$$

The $\delta f_{1\left(k-k^{\prime}-k^{\prime \prime}\right)}$ and $\delta f_{1 k^{\prime \prime}}$ in Eq. (31) and $\delta f_{1\left(k^{\prime}-k^{\prime \prime}\right)}$ in Eq. (32) can be obtained in terms of $f_{0}$ by utilizing Eq. (26)

$$
\begin{aligned}
& \delta f_{1\left(k-k^{\prime}-k^{\prime \prime}\right)}= u_{\|} \frac{e A_{\| k-k^{\prime}-k^{\prime \prime}}}{T_{\mathrm{e}}} \\
& \times \frac{\omega_{k-k^{\prime}-k^{\prime \prime}}-\omega_{* e k-k^{\prime}-k^{\prime \prime}}(u)}{\omega_{k-k^{\prime}-k^{\prime \prime}}-\omega_{\operatorname{De} k-k^{\prime}-k^{\prime \prime}}\left(u_{\|}^{2}, u_{\perp}^{2}\right)+i \nu(u)} f_{0} \\
& \delta f_{1 k^{\prime \prime}}=u_{\|} \frac{e A_{\| k^{\prime \prime}}}{T_{\mathrm{e}}} \frac{\omega_{k^{\prime \prime}}-\omega_{* \mathrm{e} k^{\prime \prime}}(u)}{\omega_{k^{\prime \prime}}-\omega_{\operatorname{De} k^{\prime \prime}}\left(u_{\|}^{2}, u_{\perp}^{2}\right)+i \nu(u)} f_{0}
\end{aligned}
$$

and

$$
\delta f_{1 k^{\prime}-k^{\prime \prime}}=u_{\|} \frac{e A_{\| k^{\prime}-k^{\prime \prime}}}{T_{\mathrm{e}}} \frac{\omega_{k^{\prime}-k^{\prime \prime}}-\omega_{* \mathrm{e} k^{\prime}-k^{\prime \prime}}(u)}{\omega_{k^{\prime}-k^{\prime \prime}}-\omega_{\operatorname{De} k^{\prime}-k^{\prime \prime}}\left(u_{\|}^{2}, u_{\perp}^{2}\right)+i \nu(u)} f_{0} .
$$

With the values of Eqs. (31)-(35) substituted into Eq. (30) and with the choice $k^{\prime \prime}=-k^{\prime}$, the ponderomotive term $\delta B^{2}$ can be produced. This choice will provide the fundamental factor $A_{\| k}$. After extracting $A_{\| k}$ factor and taking the sum of the other factors, one finds that $\delta f_{3 k}$ can be written as

$\delta f_{3 k}=u_{\|}^{3} \frac{e A_{\| k}}{T_{\mathrm{e}}} \frac{\omega-\omega_{* \mathrm{e}}(u)}{\omega-\omega_{\mathrm{De}}+i \nu(u)} \sum_{k^{\prime}} \frac{\frac{\delta \mathbf{B}_{k^{\prime}}}{B} \cdot\left(\mathbf{k}-\mathbf{k}^{\prime}\right)}{\omega_{k^{\prime}-k}-\omega_{\mathrm{Dek}^{\prime}-k}+i \nu(u)}$

$$
\times \frac{\frac{\delta \mathbf{B}_{k \prime}^{*}}{B} \cdot \mathbf{k}}{\omega_{k-k^{\prime}}-\omega_{\text {Dek-k' }}+i \nu(u)} f_{0} .
$$

It is reasonable to assume that $\left\langle\left|\delta B_{k \prime}^{2} k k^{\prime}\right|\right\rangle$ will average out on summation over $k^{\prime}$, hence $\delta f_{3 k}$ will take the form

$$
\begin{aligned}
\delta f_{3 k}= & u_{\|} \frac{e A_{\| k}}{T_{\mathrm{e}}} \frac{\omega-\omega_{* \mathrm{e}}(u)}{\omega-\omega_{\mathrm{De}}+i \nu(u)} \sum_{k^{\prime}} \frac{\left|\frac{\delta \mathbf{B}_{k \prime}}{B} \cdot \mathbf{k} u_{\|}\right|^{2}}{\omega_{k^{\prime}-k}-\omega_{\mathrm{Dek}^{\prime}-\mathrm{k}}+i \nu(u)} \\
& \times \frac{1}{\omega_{k-k^{\prime}}-\omega_{\mathrm{Dek}-\mathrm{k}^{\prime}}+i \nu(u)} f_{0} .
\end{aligned}
$$

This result for $\delta f_{3 k}$ will be used for the calculation of perturbed parallel current.

\section{Calculation of parallel current}

The parallel current associated with $\delta f_{3 k}$ is given by

$$
\begin{aligned}
j_{\| 3}= & -e \int u_{\|} \delta f_{3 k} d u \\
= & -\frac{e^{2} A_{\| k}}{T_{\mathrm{e}}} \int d u u_{\|}^{2} \frac{\omega-\omega_{* \mathrm{e}}\left[1+\eta_{\mathrm{e}}\left(\frac{m u^{2}}{2 T_{\mathrm{e}}}-\frac{3}{2}\right)\right]}{\omega-\omega_{\mathrm{De}}+i \nu(u)} \\
& \times \sum_{k^{\prime}} \frac{\left|\frac{\delta \mathbf{B}_{k^{\prime}}}{B} \cdot \mathbf{k} u_{\|}\right|^{2}}{\omega_{k^{\prime}-k}-\omega_{\mathrm{Dek}^{\prime}-\mathrm{k}}+i \nu(u)} \\
& \times \frac{1}{\omega_{k-k^{\prime}}-\omega_{\text {Dek }-\mathrm{k}^{\prime}}+i \nu(u)} f_{0} .
\end{aligned}
$$

In analogy with the linear calculation (see, e.g., Eqs. (3) and (5)), the velocity dependence of the collision frequency, in combination with $u_{\|}^{4}$, and the velocity dependence proportional to $\eta_{\mathrm{e}}$ will result in terms with a lower power of collision frequency in the denominator. This is because the $u_{\|}^{4}$ in the numerator will give more weight to larger velocities. Thus, the trial dependence is introduced

$$
\begin{aligned}
j_{\| 3}= & -\alpha_{1} A_{\| k} \varepsilon_{0} \omega_{\mathrm{pe}}^{2} \frac{\eta_{\mathrm{e}} \omega_{* \mathrm{e}}}{\omega-\omega_{\mathrm{De}}} \sum_{k^{\prime}} \frac{\left|\frac{\delta \mathbf{B}_{k^{\prime}}}{B} \cdot \mathbf{k} u_{\mathrm{th}}\right|^{2}}{\omega_{k^{\prime}-k}-\omega_{\mathrm{Dek}^{\prime}-\mathrm{k}}} \\
& \times \frac{1}{\omega_{k-k^{\prime}}-\omega_{\text {Dek }-\mathrm{k}^{\prime}}+i \nu_{\mathrm{ei}}} .
\end{aligned}
$$

Here, $\alpha_{1}$ is a free parameter for the nonlinear terms similar to $\alpha_{0}$ for the linear terms in Eq. (5). Now the contributions to the parallel current are combined as

$$
j_{\|}=j_{\| 0}+j_{\| 1}+j_{\| \phi}+j_{\| 3},
$$

and the total current is obtained as 


$$
\begin{aligned}
j_{\|}= & -\varepsilon_{0} \omega_{\mathrm{pe}}^{2}\left[\frac{\omega-\omega_{* \mathrm{e}}\left(1+\eta_{\mathrm{e}}\right)}{\omega-\omega_{\mathrm{De}}}+\alpha_{0} \frac{\eta_{\mathrm{e}} \omega_{* e}}{\omega-\omega_{\mathrm{De}}+i \nu_{\mathrm{ei}}}\right. \\
& \left.+\frac{\omega_{* \mathrm{e}}-\omega_{D e}}{\omega-\omega_{\mathrm{De}}+i \nu_{\mathrm{ei}}} \frac{k_{\|}^{2} u_{A}^{2}}{\omega\left(\omega-\omega_{\mathrm{De}}\right)}\right] A_{\|} \\
& -\alpha_{1} A_{\|} \varepsilon_{0} \omega_{\mathrm{pe}}^{2} \sum_{k^{\prime}} \frac{\eta_{\mathrm{e}} \omega_{* \mathrm{e}}}{\omega-\omega_{\mathrm{De}}} \frac{\frac{1}{2}\left|\frac{\delta \mathbf{B}_{k^{\prime}}}{B} \cdot \mathbf{k} u_{\mathrm{th}}\right|^{2}}{\omega_{k^{\prime}-k}-\omega_{\mathrm{Dek}^{\prime}-\mathrm{k}}} \\
& \times \frac{1}{\omega_{k-k^{\prime}}-\omega_{\mathrm{Dek}-\mathrm{k}^{\prime}}+i \nu_{\mathrm{ei}}}+\left(k \Longleftrightarrow k^{\prime}\right) .
\end{aligned}
$$

Note that the term proportional to $k_{\|}^{2} u_{A}^{2}$ is taken from the fluid derivation of parallel current, $j_{\| \phi}$ shown in Eq. (23). The contribution of parallel current associated with this term is due to the presence of potential perturbation in the plasma. The value of $k_{\|}$is required to be able to solve the microtearing mode dispersion relation presented in Section IV D.

\section{Determination of $\boldsymbol{k}_{\|}$required in the solution of dispersion relation}

The magnitude of $k_{\|}$can be expressed in terms of the width of the current sheet associated with the tearing mode. The estimation of $k_{\|}$presented below is based on the procedure outlined in Ref. 17 where $k_{\|}$is expressed in terms of the width of the current sheet and the magnetic shear length, that is,

$$
k_{\|}=\frac{k_{y} d}{L_{\mathrm{s}}},
$$

where $d$ is the width of the current sheet and $L_{\mathrm{s}}$ is the magnetic shear length

$$
L_{\mathrm{s}}=\frac{R q}{\hat{s}}, \quad \text { where } \quad \hat{s}=\frac{r}{q} \frac{d q}{d r} .
$$

$\mathrm{R}$ is the plasma major radius, $\hat{s}$ is the magnetic shear, $q$ is the safety factor, and $\mathrm{r}$ is the local plasma radius.

In order to estimate the value of $k_{\|}$, an estimate of the width of the current sheet is also required. Ampéres law indicates that the electrons respond to a magnetic perturbation. As shown in Ref. 19, integrating Ampéres law over the current sheet width yields

$$
\triangle^{\prime} A_{\|}=-\mu_{0} j_{\|} d
$$

where $\triangle^{\prime}$, the tearing mode stability parameter, can be expressed in terms of the poloidal mode number

$$
\triangle^{\prime}=-2 k_{y} .
$$

Note that the tearing mode stability parameter is negative (stable) for microtearing modes. In order to solve Eq. (44) for the width $d$, an estimate of the value of $j_{\|}$is also required. This can be obtained from Ampéres law

$$
j_{\|}=-\frac{1}{\mu_{0}} \frac{\partial}{\partial x^{2}} A_{\|} \approx \frac{k_{x}^{2}}{\mu_{0}} A_{\|} .
$$

The result for the current sheet width is obtained from Eqs. (44)-(46) and substituted in Eq. (42), yielding the estimate for $k_{\|}$

$$
k_{\|}=\frac{2}{L_{s}} \frac{k_{y}^{2}}{k_{x}^{2}} .
$$

\section{NONLINEAR MICROTEARING DISPERSION RELATION}

The nonlinear microtearing dispersion relation is obtained by combining Eqs. (8) (obtained using Ampéres law) and (41)

$$
\begin{aligned}
\omega[1 & \left.+\frac{k_{\perp}^{2} c^{2}}{\omega_{\mathrm{pe}}^{2}}\left(1-\frac{\omega_{\mathrm{De}}}{\omega}\right)\right] \\
= & \omega_{* \mathrm{e}}\left(1+\eta_{\mathrm{e}}\right)-\alpha_{0} \frac{\eta_{\mathrm{e}} \omega_{* e}\left(\omega-\omega_{\mathrm{De}}\right)}{\omega-\omega_{\mathrm{De}}+i \nu_{\mathrm{ei}}} \\
& -\frac{\omega_{* \mathrm{e}}-\omega_{\mathrm{De}}}{\omega-\omega_{\mathrm{De}}+i \nu_{\mathrm{ei}}} \frac{k_{\|}^{2} u_{A}^{2}}{\omega}-\alpha_{1} \eta_{\mathrm{e}} \sum_{k^{\prime}} \frac{\omega_{* \mathrm{e}}}{\omega_{k^{\prime}-k}-\omega_{\mathrm{Dek}^{\prime}-\mathrm{k}}} \\
& \times \frac{\frac{1}{2}\left|\frac{\delta \mathbf{B}_{k^{\prime}}}{B} \cdot \mathbf{k} u_{\mathrm{th}}\right|^{2}}{\omega_{k-k^{\prime}}-\omega_{\mathrm{Dek}-\mathrm{k}^{\prime}}+i \nu_{\mathrm{ei}}}+\left(k \Longleftrightarrow k^{\prime}\right) .
\end{aligned}
$$

The resonance condition,

$$
\omega_{k-k^{\prime}}=\omega_{k}-\omega_{k^{\prime}}=\omega-\omega^{\prime},
$$

and the summation over $k^{\prime}$ yields a term $-k^{\prime}$, which gives $\omega+\omega^{\prime}$. Thus, Eq. (48) can be written as

$$
\begin{aligned}
\omega[1 & \left.+\frac{k_{\perp}^{2} c^{2}}{\omega_{\mathrm{pe}}^{2}}\left(1-\frac{\omega_{\mathrm{De}}}{\omega}\right)\right] \\
= & \omega_{* \mathrm{e}}\left(1+\eta_{\mathrm{e}}\right)-\alpha_{0} \frac{\eta_{\mathrm{e}} \omega_{* e}\left(\omega-\omega_{\mathrm{De}}\right)}{\omega-\omega_{\mathrm{De}}+i \nu_{\mathrm{ei}}} \\
& -\frac{\omega_{* \mathrm{e}}-\omega_{\mathrm{De}}}{\omega-\omega_{\mathrm{De}}+i \nu_{\mathrm{ei}}} \frac{k_{\|}^{2} u_{A}^{2}}{\omega}-\alpha_{1} \eta_{\mathrm{e}} \sum_{k^{\prime}} \frac{\omega_{* \mathrm{e}}-\omega_{* \mathrm{e}}^{\prime}}{\omega_{k^{\prime}+k}-\omega_{\mathrm{Dek}^{\prime}+\mathrm{k}}} \\
& \times \frac{\frac{1}{2}\left|\frac{\delta \mathbf{B}_{k^{\prime}}}{B} \cdot \mathbf{k} u_{\mathrm{th}}\right|^{2}}{\omega_{k+k^{\prime}}-\omega_{\mathrm{Dek}+\mathrm{k}^{\prime}}+i \nu_{\mathrm{ei}}} .
\end{aligned}
$$

There are three significant additions to the previous model: (1) As a consequence of the inclusion of $\nu_{\mathrm{ei}}$ in the denominators on the right side of Eq. (50), the new MTM model is applicable for collisionless and semi-collisional regimes in addition to the collisional regime available in the earlier model. ${ }^{16}$ (2) Electrostatic potential perturbation effects are taken into account in the third term in the right hand side of Eq. (50). As a result, the dependence of MTM growth rate on density gradients, magnetic- $q$, and magnetic shear can be studied. It is anticipated that the electrostatic contribution will be important in the semi-collisional regime. (3) Toroidal effects enter the MTM dispersion relation through the inclusion of the magnetic drift frequency, $\omega_{\text {De }}$, in Eq. (50). Consequently, with the new MTM model, it is possible to determine the conditions under which the toroidal effects are important.

In Subsection V A, the dispersion relation is examined in the limit of $\nu_{\mathrm{ei}} \gg \omega$ in order to compute $\alpha_{1}$ by comparing 
the nonlinear in Eq. (50) with the nonlinear term obtained in Ref. 16.

\section{A. High collisionality limit, $v_{\mathrm{ei}} \gg \omega$}

In the collision dominant limit, nonlinear microtearing modes dispersion relation takes the form

$$
\begin{aligned}
\omega[1 & \left.+\frac{k_{\perp}^{2} c^{2}}{\omega_{\mathrm{pe}}^{2}}\left(1-\frac{\omega_{\mathrm{De}}}{\omega}\right)\right] \\
= & \omega_{* \mathrm{e}}\left(1+\eta_{\mathrm{e}}\right)+i \alpha_{0} \frac{\eta_{\mathrm{e}} \omega_{* e}\left(\omega-\omega_{\mathrm{De}}\right)}{\nu_{\mathrm{ei}}}+i \frac{\omega_{* \mathrm{e}}-\omega_{\mathrm{De}}}{\nu_{\mathrm{ei}}} \frac{k_{\|}^{2} u_{A}^{2}}{\omega} \\
& \quad-i \alpha_{1} \eta_{\mathrm{e}} \frac{u_{\mathrm{th}}^{2}}{\nu_{\mathrm{ei}}} \sum_{k^{\prime}} \frac{1}{2}\left|\frac{\delta \mathbf{B}_{k^{\prime}}}{B} \cdot \mathbf{k}\right|^{2} \frac{\omega_{* \mathrm{e}}-\omega_{* \mathrm{e}}^{\prime}}{\omega_{k}-\omega_{\mathrm{Dek}}+\omega_{k^{\prime}}-\omega_{\mathrm{Dek}^{\prime}}} .
\end{aligned}
$$

In the limit $\left(\omega_{\mathrm{De}}=0\right.$ and $\left.k_{\|}=0\right)$, the fluid dispersion in Eq. (51) is similar to the kinetic dispersion given in Eq. (8) in Ref. 16. Thus, the parameter $\alpha_{1}$ can be obtained by comparing Eq. (51) with Eq. (8) in Ref. 16 yielding the result

$$
\alpha_{1}=\frac{5}{6} \Gamma\left(\frac{11}{2}\right) .
$$

Note that the value of $\alpha_{1}$ is based on the choice of the Lorentz collision model used in Ref. 16. The numerical value of $\alpha_{1}$ depends on the choice of collision model and will change when different collision models are employed as shown in Ref. 20.

\section{B. Time variation microtearing envelope equation}

The linear growth rate will dominate initially for small initial amplitude, but it will saturate for sufficiently large amplitude. An approach is required to determine the saturation level. This can be accomplished by examining the microtearing mode envelope equation.

Express the vector potential in the following form:

$$
A_{k}(t)=\hat{A}_{k}(t) e^{-i \omega_{L} t}+c . c .
$$

where

$$
\omega_{\mathrm{L}}=\omega_{* e}\left(1+\eta_{\mathrm{e}}\right)+i \frac{\omega_{* e}-\omega_{\mathrm{De}}}{\nu_{\mathrm{ei}}} \frac{k_{\|}^{2} u_{A}^{2}}{\omega},
$$

and ignore the skin depth term $\left(k_{\perp}^{2} c^{2} / \omega_{\text {pe }}^{2}\right)$ in the collision dominant microtearing mode dispersion relation Eq. (51). The envelope equation is obtained by multiplying Eq. (51) by $A_{k}(t)$ given in Eq. (53):

$$
\begin{aligned}
\frac{\partial \hat{A}}{\partial t}= & \frac{3}{8} \Gamma\left(\frac{9}{2}\right) \frac{\eta_{\mathrm{e}} \omega_{* e}\left(\omega-\omega_{\mathrm{De}}\right)}{\nu_{\mathrm{ei}}} \hat{A}-\frac{5}{6} \Gamma\left(\frac{11}{2}\right) \eta_{\mathrm{e}} \frac{u_{\mathrm{th}}^{2}}{\nu_{\mathrm{ei}}} \\
& \times \sum_{k^{\prime}}\left|\frac{\delta \mathbf{B}_{k^{\prime}}}{B} \cdot \mathbf{k}\right|^{2} \frac{\omega_{* \mathrm{e}}-\omega_{* \mathrm{e}}^{\prime}}{\omega_{k}-\omega_{\text {Dek }}+\omega_{k^{\prime}}-\omega_{\text {Dek }^{\prime}}} \hat{A},
\end{aligned}
$$

where

$$
\delta \mathbf{B}_{k^{\prime}}=i k^{\prime} \times \hat{A}_{k^{\prime}}(t) e^{-i \omega_{\mathrm{L}} \mathrm{t}}
$$

The last term in Eq. (55) is a cubic nonlinear term, which enters as a nonlinear frequency shift. The harmonic oscillations cancel due to the term proportional to $\left|\delta \mathbf{B}_{k^{\prime}} \cdot \mathbf{k}\right|^{2}$. Therefore, Eq. (55) is a nonlinear microtearing dispersion relation as an envelope equation in time. The envelope equation can be used to calculate the saturation level of the microtearing instability. The linear growth rate will dominate initially for small initial amplitude, but it will saturate for sufficiently large amplitude. Therefore, the solution of Eq. (55) will give the initial linear growth and the final stabilization. In order to evaluate Eq. (55), in principle, the spectrum of magnetic fluctuations is needed. However, similar to the treatment of drift waves, the use of the linear fastest growing mode for microtearing modes should be sufficient.

The nonlinear term both in Eq. (50) and in Eq. (51) scales as $k^{2} \chi_{\mathrm{e}}$ where $\chi_{\mathrm{e}}$, the electron thermal diffusivity due to microtearing mode instabilities and is given by

$$
\chi_{\mathrm{e}}=\frac{u_{\mathrm{th}}^{2}}{\nu_{\mathrm{ei}}} \frac{|\delta B|^{2}}{B^{2}} .
$$

The $|\delta B|^{2} / B^{2}$ in Eq. (57) can be computed using the nonlinear microtearing envelope equation, Eq. (51). Note that in Ref. $16,|\delta B| / B$ is approximated as $\rho_{\mathrm{e}} / L_{\mathrm{Te}}$. However, the computation of $|\delta B| / B$ in Eq. (51) will depend upon the most unstable eigenvalues and as well as their sidebands in the $k_{\mathrm{y}}$ spectrum.

\section{SUMMARY}

Microtearing modes (MTMs) have been identified as a source of significant electron thermal transport in high beta tokamak discharges. A model for MTMs that can be installed in the integrated whole device predictive modeling codes is needed in order to improve the prediction of electron thermal transport and, consequently, the evolution of the plasma in devices in which MTMs have a significant role. A unified fluid/kinetic approach is used in the development of a nonlinear MTM model.

General collisional effects are included in the derivation of the MTM model. Consequently, the MTM results are applicable for collisionless, semi-collisional, and collisional regimes. The $k_{\|}$effects due to the inclusion of electrostatic potential perturbation can become important for tokamak regimes where the electron temperature gradient and magnetic- $q$ values are small, and density gradient and magnetic shear are large. The toroidal effects enter in the MTM dispersion relation through the magnetic drift, $\omega_{\text {De }}$. The magnetic drift could be important when the average curvature is important. This is when the inverse aspect ratio is comparatively large. Numerical studies carried out as a component of the ongoing research activity will aid in quantifying the parameter regimes and level of fluctuations where the toroidal effects, the electrostatic perturbation effects, and the effects of collisions of arbitrary magnitude become important.

The derived MTM dispersion relation includes the effects of electrostatic and magnetic fluctuations, collisionality, electron temperature and density gradients, arbitrary curvature, and the effects associated with parallel propagation 
vector. The derivation utilizes the gyrokinetic equation for electrons with collisions included. In the collision dominated limit, the linear dispersion relation is compared with the kinetic dispersion relation.

The electron momentum, electron density, Maxwell equations, Ampere's law, and quasi-neutrality condition are used in the derivation of the nonlinear parallel current. An iterative nonlinear approach is used to calculate distribution function, which is then used to calculate the nonlinear parallel current and nonlinear dispersion relation. The second order phase dependent nonlinearities are ignored. The third order phase independent nonlinear effects are included in the development of microtearing mode dispersion relation, and the influence of third order effects on a multi-wave system is considered. For the evolution of the nonlinear microtearing instability in time, the third order nonlinearity results in phase independent nonlinear frequency shifts which provide the saturation of the microtearing instability. An envelope equation for the nonlinear microtearing modes in the collision dominant limit is introduced. In the limit of slab geometry, absence of parallel propagation and strong collisionality, the fluid dispersion relation for nonlinear microtearing modes is found to agree with the kinetic dispersion relation obtained by Drake et al. in Ref. 16.

\section{ACKNOWLEDGMENTS}

This work was supported by the U.S. Department of Energy, Office of Science, under Award Nos. DESC0013977, DEFG02-92-ER54141, and DE-SC0012174.
${ }^{1}$ K. Wong, S. Kaye, D. Mikkelsen, J. Krommes, K. Hill, R. Bell, and B. LeBlank, Phys. Rev. Lett. 99, 135003 (2007).

${ }^{2}$ D. Applegate, C. Roach, J. Connor, S. Cowley, W. Dorland, R. Hastie, and N. Joiner, Plasma Phys. Controlled Fusion 49, 1113 (2007).

${ }^{3}$ W. Guttenfelder, J. Candy, S. M. Kaye, W. M. Nevins, E. Wang, R. E. Bell, G. W. Hammett, B. P. LeBlanc, D. R. Mikkelsen, and H. Yuh, Phys. Rev. Lett. 106, 155004 (2011).

${ }^{4}$ W. Guttenfelder, J. Candy, S. M. Kaye, W. M. Nevins, E. Wang, J. Zhang, R. E. Bell, N. A. Crocker, G. W. Hammett, B. P. LeBlanc, D. R. Mikkelsen, Y. Ren, and H. Yuh, Phys. Plasmas 19, 056119 (2012).

${ }^{5}$ S. M. Kaye, W. Guttenfelder, R. E. Bell, S. P. Gerhardt, B. P. LeBlanc, and R. Maingi, Phys. Plasmas 21, 082510 (2014).

${ }^{6}$ J. D. Callen, Phys. Rev. Lett. 39, 1540 (1977).

${ }^{7}$ R. D. Hazeltine, D. Dobrott, and T. S. Wang, Phys. Fluids 18, 1778 (1975).

${ }^{8}$ P. H. Rebut, P. P. Lallia, and M. L. Watkins, in Proceedings of the 12 th IAEA Conference on Plasma Physics and Controlled Nuclear Fusion Research Nice, France (1988), Paper No. IAEA-CN-50/D-4-1.

${ }^{9}$ D. Dickinson, C. M. Roach, S. Saarelma, R. Scannell, A. Kirk, and H. R. Wilson, Phys. Rev. Lett. 108, 135002 (2012).

${ }^{10}$ J. Weiland and C. S. Liu, J. Plasma Phys. 79, 1015 (2013).

${ }^{11}$ N. Ohyabu, G. Jahns, R. Stambaugh, and E. Strait, Phys. Rev. Lett. 58, 120 (1987).

${ }^{12}$ J. Kesner and S. Migliuolo, Nucl. Fusion 39, 163 (1999).

${ }^{13}$ S. Moradi, I. Pusztai, W. Guttenfelder, T. Fülöp, and A. Mollén, Nucl. Fusion 53, 063025 (2013).

${ }^{14}$ I. Predebon and F. Sattin, Phys. Plasmas 20, 040701 (2013).

${ }^{15}$ T. Rafiq, A. H. Kritz, J. Weiland, A. Y. Pankin, and L. Luo, Phys. Plasmas 20, 032506 (2013).

${ }^{16}$ J. F. Drake, N. T. Gladd, C. S. Liu, and C. L. Chang, Phys. Rev. Lett. 44, 994 (1980).

${ }^{17} \mathrm{~J}$. Weiland, Stability and Transport in Magnetic Confinement System, Springer Series on Atomic, Optical and Plasma Physics Vol. 71 (Springer, 2012).

${ }^{18} \mathrm{~J}$. Nilsson and J. Weiland, Nucl. Fusion 34, 803 (1994).

${ }^{19}$ N. T. Gladd, J. F. Drake, C. L. Chang, and C. S. Liu, Phys. Fluids 23, 1182 (1980).

${ }^{20}$ A. B. Hassam, Phys. Fluids 23, 38 (1980). 